\title{
Long QT syndrome: novel insights into the mechanisms of cardiac arrhythmias
}

\author{
Robert S. Kass ${ }^{1}$ and Arthur J. Moss ${ }^{2}$ \\ ${ }^{1}$ Department of Pharmacology, Columbia University College of Physicians and Surgeons, New York, New York, USA \\ ${ }^{2}$ Department of Medicine, Heart Research Follow-up Program, University of Rochester School of Medicine and Dentistry, \\ Rochester, New York, USA
}

The congenital long QT syndrome is a rare disorder in which mutation carriers are at risk for polymorphic ventricular tachycardia and/or sudden cardiac death. Discovery and analysis of gene mutations associated with variants of this disorder have provided novel insight into mechanisms of cardiac arrhythmia and have raised the possibility of mutation-specific therapeutic intervention.

J. Clin. Invest. 112:810-815 (2003). doi:10.1172/JCI200319844.

\section{Historical perspective}

In 1957, Jervell and Lange-Nielsen first described a family with long QT syndrome (LQTS) (1). The family consisted of unrelated parents and their six children, four of whom were deaf with frequent fainting attacks precipitated by acute emotional arousal and exercise. Three of the four deaf children died suddenly while playing at the ages of 4, 5, and 9 years. An ECG was never recorded for the first deceased deaf child; however, ECGs of the remaining deaf children indicated markedly prolonged QT intervals (Figure 1). Both parents and the other two children were healthy, with normal hearing and normal ECGs. In 1958, Levine and Woodworth (2) reported the sudden death of a 13-yearold deaf boy with recurrent syncope and QT prolongation. Both parents were normal with no consanguinity. This combination of congenital deafness, QT prolongation, and recurrent syncope in five children from two independent families with normal parents was initially interpreted as a condition characterized by an autosomal recessive mode of inheritance.

In 1963 and 1964, Romano et al. (3) and Ward (4) respectively, reported separate families with QT prolongation in one parent and several children, all of whom possessed normal hearing but experienced recurrent syncope and sudden death. The pattern of symptoms and ECG findings in these two families support-

\footnotetext{
Address correspondence to: Robert S. Kass, Department of Pharmacology, Columbia University College of Physicians and Surgeons, 630 West 168th Street, P\&S 7-401, New York, New York 10032, USA. Phone: (212) 305-7444;

Fax: (212) 342-2703; E-mail: rsk20@columbia.edu.

Conflict of interest: The authors have declared that no conflict of interest exists.

Nonstandard abbreviations used: long QT syndrome (LQTS); heart rate-corrected QT (QTc); sympathetic nervous system (SNS); protein kinase A (PKA); protein phosphatase 1 (PP1); $\beta$-adrenergic receptor ( $\beta$-AR).
}

ed the presence of a condition characterized by an autosomal dominant mode of inheritance.

During the 1960s and 1970s, a number of individuals and families were reported with clinical features similar to those described by Jervell and Lange-Nielsen (1) or Romano et al. (3) and Ward (4). In 1979 Moss and Schwartz established the prospective International LQTS Registry for enrollment and follow-up of proband-identified LQTS families in order to determine the clinical course and hereditary features of LQTS (5). During the past 24 years, the Registry has enrolled over 1,200 LQTS families and has provided an understanding of the diagnostic features of this disorder, the natural history of the condition, and the efficacy of various therapies. In addition, the Registry has been an invaluable resource in the identification of genetic mutations that cause this inherited disorder.

\section{Disease diagnosis}

LQTS occurs infrequently in the general population with an estimated frequency of about 1 in 5,000 people. Patients with LQTS are usually identified by QT prolongation on the ECG during clinical evaluation of unexplained syncope, as part of a family study when one family member has been identified with the syndrome, or in the investigation of patients with congenital neural deafness. Clinical criteria have been developed to determine the probability that a patient has LQTS (Table 1). The two most important diagnostic features of LQTS are prolongation of the heart rate-corrected QT interval (QTc $\geq$ 0.46 seconds) and stress-induced syncope.

The syncope that occurs in this disorder is due to a transient, rapid, polymorphic ventricular tachycardia described as torsade de pointes (twisting of the points) that is associated with the underlying delayed ventricular repolarization manifest on the ECG as QTc prolongation (Figure 1d). Sudden death occurs when a poly- 
b

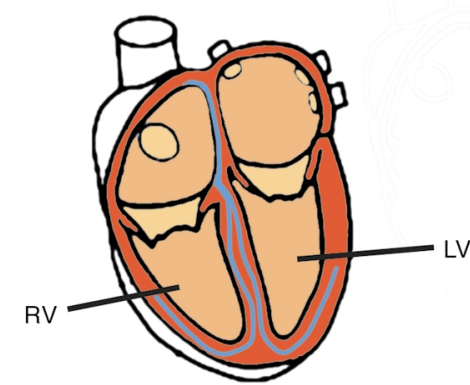

C

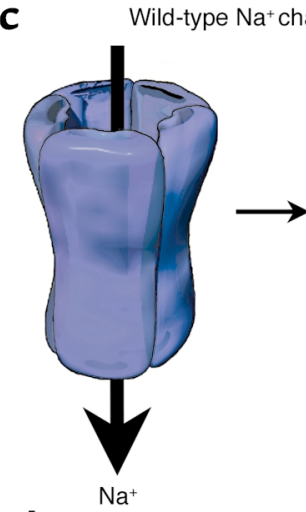

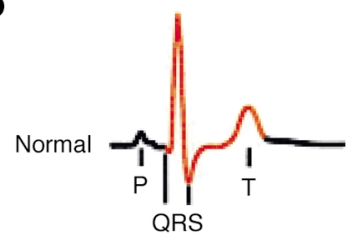

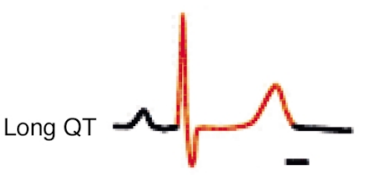

Normal d

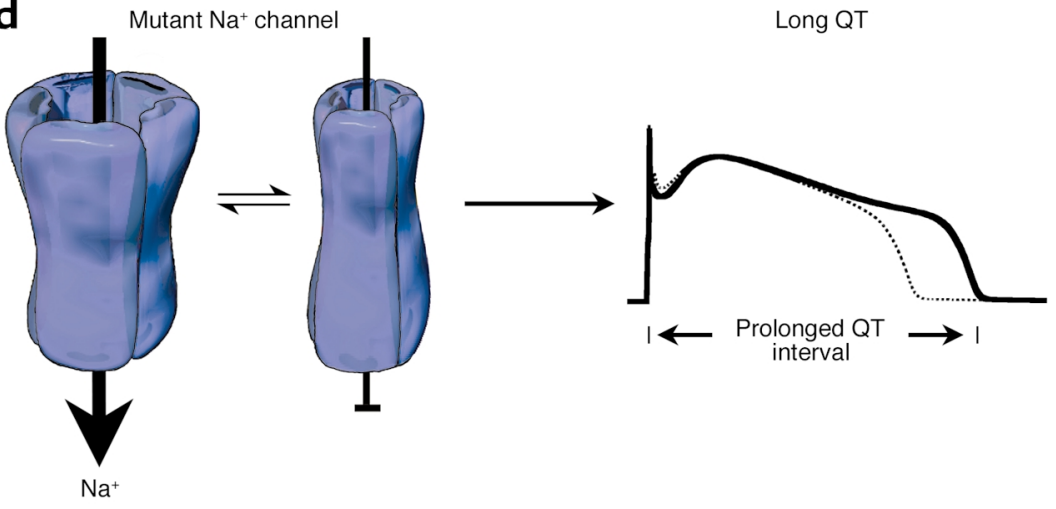

\section{Figure 1}

Mutation-altered $\mathrm{Na}^{+}$channel inactivation underlies the LQT-3 phenotype. (a) Schematic view of the human heart emphasizing right ventricle (RV) and left ventricle (LV), which contain the substrates for altered electrical signaling in LQTS. (b) The clinical phenotype of LQTS is a prolonged QT interval of the ECG. (c) Gating of normal (wild-type) human heart $\mathrm{Na}^{+}$channels during the action potential plateau. Channels rapidly enter an absorbing inactivated state from which they rarely reopen, and thus the QT interval (far right) is not greatly affected by wild-type $\mathrm{Na}^{+}$channels. (d) $\mathrm{Na}^{+}$channel gating in most forms of LQT-3 syndrome is altered. During the plateau phase, channels enter a mode of gating in which inactivation is no longer an absorbing state. A small fraction of channels open, enter a nonconducting inactivated state, and then transition back and forth between open and nonconducting states for the duration of the action potential plateau. This produces a small depolarizing current that prolongs the QT interval of the ECG of mutation carriers (far right). Modified with permission from refs. 25 and 26 .

state, often referred to as the plateau phase of the ventricular action potential, is not only cardioprotective against premature excitation but is also essential to maintaining the proper timing between diastolic filling and ejection intervals. It is the plateau phase of the ventricular action potential that thus determines the QT interval of the ECG. Over 50 years ago, Silvio Weidmann (9) discovered that this crucial plateau phase is maintained by a del-

morphic ventricular tachycardia episode deteriorates into ventricular fibrillation. Syncope and sudden death are most frequent in childhood and adolescence. The risk of cardiac events is higher in males before puberty and higher in females during adulthood $(6,7)$. The clinical course of patients with LQTS is quite variable and is influenced by the length of the QTc interval, gender, environmental factors, genotype, and therapy (8).

\section{Mutation-induced ion channel dysfunction and LQTS}

The ventricular action potential of the human heart is distinct in that the temporal period separating excitation of ventricular cells from relaxation, or repolarization, is very long, typically on the order of 450 milliseconds. This timing is crucial because as long as the ventricular tissue is depolarized it cannot be re-excited due to the unavailability of key voltage-gated $\mathrm{Na}^{+}$channels which normally enter a nonconducting inactivated state during this period (Figure 1c). The duration of this depolarized icate balance of small ionic conductances, which, though energetically favorable, predispose this period of the electrical cycle in the heart to disturbances that may be caused by otherwise harmlessly small changes in transmembrane ionic activity.

Before genetic information was successfully used to identify genes associated with congenital LQTS, various candidate ion channels (and thus channel-coding genes) were suspected to underlie the clinical defect of delayed ventricular relaxation. Molecular genetic approaches removed at least some of the uncertainty by demonstrating a clear association between mutations in genes encoding specific ion channel subunits and LQTS (reviewed in ref. 10). This work has revealed that mutations in at least five genes coding for key cardiac $\mathrm{K}^{+}$channel subunits result in a loss or reduction of channel activity that can cause variants of LQTS (Table 2). Surprisingly, mutations in the principal $(\alpha)$ subunit of a key heart $\mathrm{Na}^{+}$channel (encoded by $S C N 5 A)$ that, in general, result in a gain of channel 
Table 1

Diagnostic criteria for long QT syndrome

\begin{tabular}{|c|c|}
\hline Features & Points \\
\hline \multicolumn{2}{|l|}{ ECG findings ${ }^{A}$} \\
\hline \multicolumn{2}{|l|}{ QTc } \\
\hline $\begin{array}{l}\geq 0.48 \mathrm{~s} \\
0.46-0.47 \mathrm{~s} \\
0.45 \mathrm{~s}\end{array}$ & $\begin{array}{l}3 \\
2 \\
1\end{array}$ \\
\hline Torsade de pointes ${ }^{B}$ & 2 \\
\hline Twave alternans & 1 \\
\hline Notched T wave in three leads & 1 \\
\hline Low heart rate for age $\mathrm{C}^{\mathrm{C}}$ & 0.5 \\
\hline \multicolumn{2}{|l|}{ Clinical History } \\
\hline \multicolumn{2}{|l|}{ Syncope ${ }^{B}$} \\
\hline $\begin{array}{l}\text { With stress } \\
\text { Without stress }\end{array}$ & $\begin{array}{l}2 \\
1\end{array}$ \\
\hline Congenital deafness & 0.5 \\
\hline \multicolumn{2}{|l|}{ Family history } \\
\hline Family members with definite LQTSD & 1 \\
\hline $\begin{array}{l}\text { Unexplained sudden cardiac death before } \\
\text { age } 30 \text { among immediate family members }\end{array}$ & 0.5 \\
\hline
\end{tabular}

Scoring: $\leq 1$ point, low probability of LQTS; $2-3$ points, intermediate probability of LATS; $\geq 4$ points, high probability of LQTS. AFindings in the absence of medications or disorders known to affect these ECG findings. QTc calculated by Bazett's formula, where QTc $=\mathrm{QT} / \sqrt{ } \mathrm{RR}$. ${ }^{\mathrm{B}}$ Mutually exclusive. ${ }^{\mathrm{C}}$ Resting heart rate below the second percentile for age. ${ }^{\mathrm{D} T h e}$ same family member cannot be counted for both of these criteria. Reprinted with permission from ref. 6 .

function also cause yet another variant of LQTS, known as LQT-3 syndrome. These discoveries have prompted at least three new areas of research that impact not only the diagnosis and management of LQTS, but also our understanding of human cardiovascular physiology. These include (a) mutation-specific therapeutic strategies (b) identification of mutation-specific risk of cardiac events, and (c) mechanistic insight into the role of altered channel function and regulation in the control of QT intervals in the heart. The first two areas of research focus will be discussed below. Here we will focus on two areas in which fundamentally novel insight into human physiology emerged from investigation of the mechanistic basis of LQTS: the role of altered $\mathrm{Na}^{+}$channel gating in control of the QT interval, and the molecular link between the sympathetic nervous system (SNS) and electrical signaling in the ventricle.

\section{$\mathrm{Na}^{+}$channels}

Expression of ion channels in heterologous systems allows for investigation of inherited ion channel defects at the single-protein and cellular levels to directly identify the disease-associated alteration in ion channel function. Disease-linked mutations provide an opportunity to understand the mechanistic basis of human disease from altered molecular function to the clinical syndrome. Initial investigation of SCN5A mutations linked to LQT-3 syndrome revealed striking mutation-induced defects in channel behavior that were consistent with the disease phenotype (11). Classically, $\mathrm{Na}^{+}$channel activation is associated with the spread of depolarization in the heart that underlies the QRS complex of the ECG. In the case of LQT-3 syndrome, however, defects in the $\mathrm{Na}^{+}$channel were linked to delay in ventricular repolarization (prolongation of the QT interval). These initial experiments clearly showed a novel mechanism that could explain this unexpected result: the inherited mutation disrupted the "inactivation" process of the channel such that, during the plateau phase of the action potential, a small number of $\mathrm{Na}^{+}$ channels do not inactivate (become nonconducting) but in fact reopen to provide a very small depolarizing current (Figure 1d). Exactly as Weidmann had predicted (9), even though this late current is only a fraction of the total $\mathrm{Na}^{+}$channel current responsible for the QRS complex, this small disease-associated perturbation in plateau current is responsible for prolonging the QT interval in mutation carriers and raising the risk of cardiac events (12). Thus, the molecular genetic analysis of LQTS patients has led to a novel understanding of the importance of $\mathrm{Na}^{+}$ channel activity in controlling not only the QRS complex, but also the duration of the ventricular electrical response: the QT interval. Recently, mutations in SCN1A, the gene coding for the human neuronal $\mathrm{Na}^{+}$channel $\alpha$ subunit associated with epilepsy, have been reported to cause similar defects in channelinactivation gating (13). As yet, the cellular consequences of such epilepsy-associated mutations remain elusive, but mechanistic insights gained from investigation of cardiac defects are very likely to have widespread implications.

\section{$\mathrm{K}^{+}$channels: insight into adrenergic control of cardiac electrical activity}

Investigation of the molecular basis of LQTS has led to fundamental insight into the molecular identity of key $\mathrm{K}^{+}$channel subunits in the heart, notably the two key delayed rectifier currents $\mathrm{I}_{\mathrm{Kr}}$ and $\mathrm{I}_{\mathrm{Ks}}$, which have been demonstrated in animal models to be crucial to control of cardiac action potential duration. In particular, mutations in KCNQ1, which codes for the $\alpha$ subunit of the $\mathrm{I}_{\mathrm{Ks}}$ channel, cause LQT-1 syndrome, and mutations in $K C N E 1$, the gene coding for the auxiliary $\beta$ subunit of the $\mathrm{I}_{\mathrm{Ks}}$ channel, cause LQT-5 syndrome (10). It had been

\section{Table 2}

Molecular and cellular mechanisms of cardiac arrhythmias

\begin{tabular}{|c|c|c|c|}
\hline Disease & Gene (alternate name) & Protein & Reference \\
\hline LQT-1 & KVLQT1 (KCNQ1) & $\mathrm{I}_{\mathrm{Ks}} \mathrm{K}^{+}$channel $\alpha$ subunit & 28 \\
\hline LQT-2 & HERG (KCNH2) & $\mathrm{I}_{\mathrm{Kr}} \mathrm{K}^{+}$channel $\alpha$ subunit & 29 \\
\hline LQT-3 & SCN5A & $\mathrm{I}_{\mathrm{Na}} \mathrm{K}^{+}$channel $\alpha$ subunit & 30 \\
\hline LQT-4 & $A N K B$ & ANKRIN- $\beta$ & 31 \\
\hline LQT-5 & $\operatorname{minK}(K C N E 1)$ & $\mathrm{I}_{\mathrm{Ks}} \mathrm{K}^{+}$channel $\beta$ subunit & 32 \\
\hline LQT-6 & MiRP1 (KCNE2) & $\mathrm{I}_{\mathrm{Kr}} \mathrm{K}^{+}$channel $\beta$ subunit & 33 \\
\hline LQT-7 & KCN/2 & $\mathrm{I}_{\mathrm{Kr}} \mathrm{K}^{+}$channel $\alpha$ subunit & 34 \\
\hline
\end{tabular}




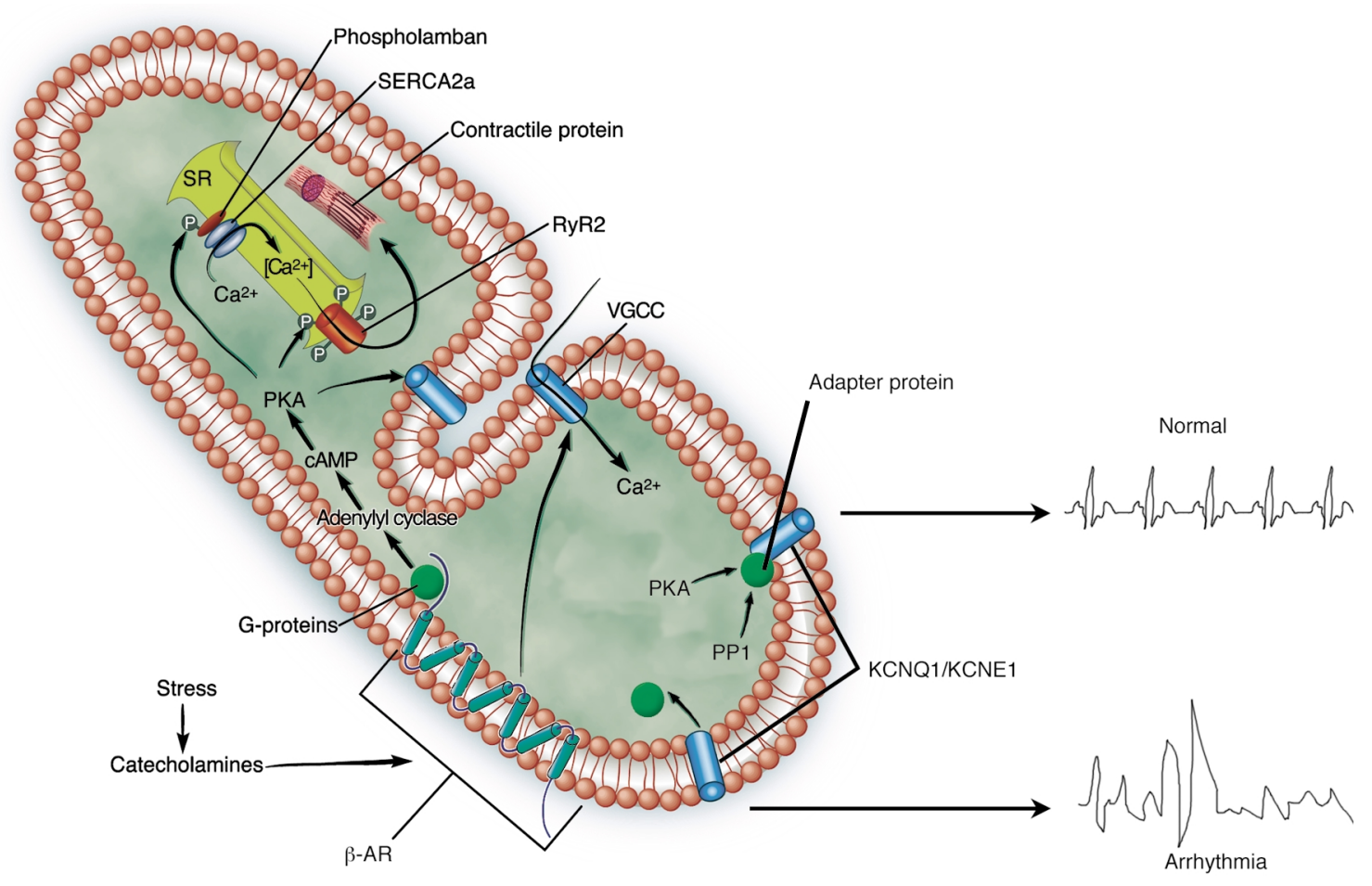

Figure 2

Disruption of local signaling domains occurs in LQT-1 syndrome. Stimulation of $\beta$-adrenergic receptors ( $\beta$-ARs) in the heart leads to PKAdependent phosphorylation of multiple intracellular targets in cardiac myocytes. These targets include the ryanodine receptor (RyR2), L-type calcium channels, and the KCNQ1/KCNE1 K+ channel. In response to stress in healthy patients, $\beta$-AR stimulation results in phosphorylation of all three of these targets and uniform electrical activity on the ECG (upper ECG). When the KCNQ1/KCNE1 complex is disrupted by an inherited mutation, an unbalanced cellular response occurs, which leads to dysfunctional rhythm (lower ECG). Modified with permission from ref. 27. SR, sarcoplasmic reticulum; VGCC, voltage-gated calcium channel.

well established in animal models that $\mathrm{I}_{\mathrm{Ks}}$ is strongly regulated by SNS stimulation, such that SNS stimulation increases reserves of repolarizing current and contributes to the shortening of action potential that occurs in parallel with the SNS stimulation-induced increases in heart rate (14). Further, clinical data indicate that carriers of mutations in either KCNQ1 or KCNE1 are at increased risk of experiencing a fatal cardiac arrhythmia in the face of elevated SNS activity (15). Together, these findings motivated investigation into molecular links between the SNS and regulation of KCNQ1/KCNE1 channels in the human heart. This work resulted in the discovery that the KCNQ1/KCNE1 channel actually forms a macromolecular signaling complex: coupled to the carboxy-terminal domain of the channel is an adaptor protein, yotiao, which in turn binds to the regulatory enzymes protein kinase $\mathrm{A}(\mathrm{PKA})$ and protein phosphatase 1 (PP1) (Figure 2) (16). Thus the channel, via the adapter protein, recruits enzymes that can upregulate (PKA) and downregulate (PP1) channel activity by phosphorylation and dephosphorylation of a serine in its amino-terminal domain (16). When this complex is disrupted, the channel is not properly regulated and there is imbalance in control of the ventricular action potential, which leads to high risk of arrhythmia. Because other targets of PKA, such as the ryanodine receptor of the sarcoplasmic reticulum, form independent macro- molecular signaling complexes, selective disruption of the KCNQ1/KCNE1 signaling complex by inherited mutations may disrupt a micromolecular signaling domain restricted to one channel - a potentially novel mechanism that may mechanistically contribute to the genesis of cardiac arrhythmias (Figure 2) (17).

\section{Clinical evaluation: phenotype can suggest genotype}

The discovery that distinct LQTS variants were associated with genes coding for different ion channel subunits has had a major impact on the diagnosis and analysis of LQTS patients. It is clear that there are distinct risk factors associated with the different LQTS genotypes, and this must be taken into account during patient evaluation and diagnosis. The greatest difference in risk factors becomes apparent in comparing LQT-3 syndrome patients (SCN5A mutations) and patients with LQT-1 syndrome (KCNQ1 mutations) or LQT-2 syndrome (HERG mutations). This was made clear in an extensive and collaborative study in which the risk of cardiac events was found to be greatest during rest, during bradycardia, or, in the case of LQT-3 syndrome patients, under conditions of low sympathetic nerve activity (15). In contrast, cardiac events in LQT-2 syndrome patients were associated with arousal and/or conditions in which patients were startled, 
whereas LQT-1 syndrome patients were found to be at greatest risk of cardiac events during exercise, conditions of elevated heart rate, and sympathetic nerve activity (15). Clearly, identifying the genotype is essential during diagnosis, as risk factors are somewhat gene specific. Some groups have begun investigating the use of exercise or stress testing to estimate the likelihood that a patient possesses a specific genotype (18). Additionally, a major effort has been made to link ECG waveform characteristics to genotype in order to estimate the genetic basis of familial LQTS in patient candidates (19). However, ultimately, genotype identification by either direct sequencing or other technologies is necessary to unequivocally determine the genetic basis of the clinical defect.

\section{Therapeutic strategy defined by genotype}

One of the major contributions of the integration of cellular, molecular, and genetic techniques in the investigation of the causes and treatment of congenital LQTS has been the emergence of mutation-specific strategies for disease therapy. Because the functional consequences of most of the $\mathrm{Na}^{+}$channel mutations that cause LQT-3 syndrome are subtle increases in channel activity during the action potential plateau, cellular experimental work suggested that conventional $\mathrm{Na}^{+}$ channel blockers such as mexiletine, tocainide, and lidocaine might prove useful in treating this LQTS variant (20). This has, in fact, proven to be the case, and other $\mathrm{Na}^{+}$channel blockers such as flecainide have emerged with great promise as therapeutic agents specific for LQT-3 syndrome patients $(21,22)$. $\beta$-blocker therapy is most effective in preventing recurrence of cardiac events and lowering the death rate in LQT-1 and LQT-2 syndrome patients but is much less effective in the treatment of LQT-3 syndrome patients $(15,23)$. $\beta$-blocking drugs are associated with a significant reduction in cardiac events in LQTS patients, even though these drugs have minimal effect on the QTc interval (23). It is believed that the efficacy of $\beta$-blocker therapy is related to an attenuation of adrenergic-mediated trigger mechanisms in the disorder. $\beta$-blockers do not provide absolute protection against fatal cardiac arrhythmias. Recent studies indicate that the implanted cardioverter defibrillator is effective in preventing sudden cardiac death in LQTS patients (24). The combination of $\beta$-blocker drugs and an implanted defibrillator is a safe and reliable form of therapy for managing high-risk LQTS. Particularly in the case of LQT-1 syndrome patients, $\beta$-blocker administration is thought to reduce incidents of cellular imbalance in response to stimulation of $\beta$-adrenergic receptors ( $\beta$-ARs) (Figure 2 ), which occurs when one target of $\beta$-AR stimulation, the KCNQ1/KCNE1 channel, is eliminated from the cellular response. In fact, because lower heart rates provoke cardiac events in LQT- 3 mutant gene carriers, $\beta$-blockade may be pro-arrhythmic in this patient group. Thus genotype, which underlies phenotype, can dictate the most promising therapeutic approach.

\section{What remains unknown and future directions}

Investigations into the clinical aspects and basic causal mechanisms of LQTS have provided novel and important insight into the fundamental nature of the electrical activity of the human heart and how small perturbations in ion flow can have important consequences for human disease. We know that there are multiple variants of the disease that are linked to mutations in specific ion channel subunit genes, and that the unifying clinical phenotype is delay of repolarization in the ventricle. Though great progress has been made, many important questions remain. What is the cellular mechanism responsible for the triggering of fatal events in the context of the whole heart? Why does repolarization delay (QT prolongation) lead to early after-depolarizations and trigger arrhythmogenic events? Does altered cellular calcium play a role in these arrhythmias? Are mutations in other ion channel genes responsible for as yet unrecognized variants of LQTS? Finally, can we identify gene variants that may predispose individuals to arrhythmia risk if, and only if, other subtle mutations and/or polymorphisms exist in another gene in the same individual? Answers to these questions with respect to LQTS will no doubt provide further insight into the fundamental mechanisms of the incredibly robust and resilient, but ever so sensitive, electrical network of the human heart.

1. Jervell, A., and Lange-Nielsen, F. 1957. Congential deafmutism, functional heart disease with prolongation of the $\mathrm{Q}$ interval, and sudden death. Am. Heart J. 54:59-68.

2. Levine, S.A., and Woodworth, C.R. 1958. Congenital deaf-mutism, prolonged Q-T interval, syncopal attacks and sudden death. N. Engl.J. Med. 259:412-417.

3. Romano, C., Gemme, G., and Pongiglione, R. 1963. Artimie cardiache rare dell'eta pediatria. Clin. Pediatr. (Phila.). 45:656-683.

4. Ward, O.C. 1964. New familial cardiac syndrome in children. J. Ir. Med. Assoc. 54:103-106.

5. Moss, A.J., and Schwartz, P.J. 1982. Delayed repolarization (QT or QTU prolongation) and malignant ventricular arrhythmias. Mod. Concepts Cardiovasc. Dis. 51:85-90.

6. Schwartz, P.J., Moss, A.J., Vincent, G.M., and Crampton, R.S. 1993. Diagnostic criteria for the long QT syndrome. An update. Circulation. 88:782-784.

7. Locati, E.H., et al. 1998. Age- and sex-related differences in clinical manifestations in patients with congenital long-QT syndrome: findings from the International LQTS Registry. Circulation. 97:2237-2244.

8. Moss, A.J., et al. 1991. The long QT syndrome. Prospective longitudinal study of 328 families. Circulation. 84:1136-1144.

9. Weidmann, S. 1951. Effect of current flow on the membrane potential of cardiac muscle. J. Physiol. 115:227-236.

10. Sanguinetti, M.C. 2000. Long QT syndrome: ionic basis and arrhythmia mechanism in long QT syndrome type 1. J. Cardiovasc. Electrophysiol. 11:710-712.

11. Bennett, P.B., Yazawa, K., Makita, N., and George, A.L. 1995. Molecular mechanism for an inherited cardiac arrhythmia. Nature. 376:683-685.

12. Clancy, C.E., and Rudy, Y. 1999. Linking a genetic defect to its cellular phenotype in a cardiac arrhythmia. Nature. 400:566-569.

13. Lossin, C., Wang, D.W., Rhodes, T.H., Vanoye, C.G., and George, A.L., Jr. 2002. Molecular basis of an inherited epilepsy. Neuron. 34:877-884.

14. Kass, R.S., and Wiegers, S.E. 1982. The ionic basis of concentration-related effects of noradrenaline on the action potential of calf cardiac purkinje fibres. J. Physiol. 322:541-558.

15. Schwartz, P.J., et al. 2001. Genotype-phenotype correlation in the longQT syndrome: gene-specific triggers for life-threatening arrhythmias. Circulation. 103:89-95.

16. Marx, S.O., et al. 2002. Requirement of a macromolecular signaling complex for beta adrenergic receptor modulation of the KCNQ1-KCNE1 potassium channel. Science. 295:496-499.

17. Kass, R.S., Kurokawa, J., Marx, S.O., and Marks, A.R. 2003. Leucine/ isoleucine zipper coordination of ion channel macromolecular signal- 
ing complexes in the heart. Roles in inherited arrhythmias. Trends Cardiovasc. Med. 13:52-56.

18. Takenaka, K., et al. 2003. Exercise stress test amplifies genotype-phenotype correlation in the LQT1 and LQT2 forms of the long-QT syndrome. Circulation. 107:838-844.

19. Moss, A.J., et al. 1995. Electrocardiographic T-wave patterns in genetically distinct forms of the hereditary long QT syndrome. Circulation. 92:2929-2934.

20. An, R.H., Bangalore, R., Rosero, S.Z., and Kass, R.S. 1996. Lidocaine block of LQT-3 mutant human Na+ channels. Circ. Res. 79:103-108.

21. Benhorin, J., et al. 2000. Effects of flecainide in patients with new SCN5A mutation: mutation-specific therapy for long-QT syndrome? Circulation. 101:1698-1706.

22. Windle, J.R., Geletka, R.C., Moss, A.J., Zareba, W., and Atkins, D.L. 2001 Normalization of ventricular repolarization with flecainide in long QT syndrome patients with SCN5A:DeltaKPQ mutation. Ann. Noninvasive. Electrocardiol. 6:153-158.

23. Moss, A.J., et al. 2000. Effectiveness and limitations of beta-blocker therapy in congenital long-QT syndrome. Circulation. 101:616-623.

24. Zareba, W., et al. 2003. Implantable cardioverter defibrillator in high-risk long QT syndrome patients. J. Cardiovasc. Electrophysiol. 14:337-341.

25. Clancy, C.E., and Kass, R.E. 2002. Defective cardiac ion channels: from mutations to clinical syndromes. J. Clin. Invest. 110:1075-1077. doi:10.1172/JCI200216945.

26. Keating, M.T., and Sanguinetti, M.C. 2001. Molecular and cellular mechanisms of cardiac arrhythmias. Cell. 104:569-580.

27. Marks, A.R. 2003. Calcium and the heart: a question of life and death. J. Clin. Invest. 111:597-600. doi:10.1172/JCI200318067.

28. Wang, Q., et al. 1996. Positional cloning of a novel potassium channel gene: KVLQT1 mutations cause cardiac arrhythmias. Nat. Genet. 12:17-23.

29. Curran, M.E., et al. 1995. A molecular basis for cardiac arrhythmia: HERG mutations cause long QT syndrome. Cell. 80:795-803.

30. Wang, Q., et al. 1995. SCN5A mutations associated with an inherited cardiac arrhythmia, long QT syndrome. Cell. 80:805-811.

31. Mohler, P.J., et al. 2003. Ankyrin-B mutation causes type 4 long-QT cardiac arrhythmia and sudden cardiac death. Nature. 421:634-639.

32. Splawski, I., Tristani-Firouzi, M., Lehmann, M.H., Sanguinetti, M.C., and Keating, M.T. 1997. Mutations in the hminK gene cause long QT syndrome and suppress IKs function. Nat. Genet. 17:338-340.

33. Abbott, G.W., et al. 1999. MiRP1 forms IKr potassium channels with HERG and is associated with cardiac arrhythmia. Cell. 97:175-187.

34. Plaster, N.M., et al. 2001. Mutations in Kir2.1 cause the developmental and episodic electrical phenotypes of Andersen's syndrome. Cell. 105:511-519. 